\title{
Lipocalin 2 as Putative Modulator of Local Inflammatory Processes in the Spinal Cord and Component of Organ Cross-talk After Spinal Cord Injury
}

\section{Victoria Behrens}

RWTH Aachen University: Rheinisch-Westfalische Technische Hochschule Aachen

\section{Clara Voelz}

RWTH Aachen University: Rheinisch-Westfalische Technische Hochschule Aachen

Nina Müller

RWTH Aachen University: Rheinisch-Westfalische Technische Hochschule Aachen

\section{Weiyi Zhao}

RWTH Aachen University: Rheinisch-Westfalische Technische Hochschule Aachen

\section{Tim Clarner}

RWTH Aachen University: Rheinisch-Westfalische Technische Hochschule Aachen

\section{Cordian Beyer}

RWTH Aachen University: Rheinisch-Westfalische Technische Hochschule Aachen

Adib Zendedel ( $\nabla$ azendedel@ukaachen.de)

Neuroanatomy https://orcid.org/0000-0001-7415-9346

\section{Research Article}

Keywords: Spinal cord, astroglia, lipocalin 2, organ-crosstalk

Posted Date: May 25th, 2021

DOI: https://doi.org/10.21203/rs.3.rs-513109/v2

License: (9) (i) This work is licensed under a Creative Commons Attribution 4.0 International License. Read Full License

Version of Record: A version of this preprint was published at Molecular Neurobiology on August $21 \mathrm{st}$, 2021. See the published version at https://doi.org/10.1007/s12035-021-02530-7. 


\section{Abstract}

Lipocalin 2 (Lcn2), an immunomodulator, regulates various cellular processes such as iron transport and defense against bacterial infection. Under pathological conditions, Lcn2 promotes neuroinflammation via the recruitment and activation of immune cells and glia, particularly microglia and astrocytes. Although it seems to have a negative influence on the functional outcome in spinal cord injury (SCl), the extent of its involvement in $\mathrm{SCl}$ and the underlying mechanisms are not yet fully known. In this study, using a $\mathrm{SCl}$ contusion mouse model, we first investigated the expression pattern of Lcn2 in different parts of the CNS (spinal cord and brain), blood serum and in the liver. Interestingly, we could note a significant increase in Lcn2 throughout the whole spinal cord, in the brain, liver and in blood serum. This demonstrates the diversity of its possible sites of action in SCl. Further, genetic deficiency of $\operatorname{Lcn} 2\left(\mathrm{Lcn}^{-1-}\right)$ significantly reduced certain aspects of gliosis in the SCl-mice. Taken together, our studies provide first valuable hints, suggesting that $\mathrm{Lcn} 2$ is involved in the local and systemic effects post $\mathrm{SCl}$, and might modulate the impairment of different peripheral organs after injury.

\section{Introduction}

Spinal cord injury (SCl) is a devastating event that causes life-long health restrictions including paralysis, loss of sensation and vegetative functions, pain, and psychological impairment [1]. Despite many efforts, there is presently no comprehensive treatment protocol available to effectively treat this injury, mainly owed to the complexity of nerve fiber tract destructions, neuronal death and poor restoration capacities of physiological function [2, 3]. In SCl, the primary injury refers to the initial physical damage of the spinal cord (SC), which is accompanied by hemorrhage, ischemia and local neuronal death, while the secondary injury phase is characterized by progressive damage of the SC, demyelination, astrogliosis and neuroinflammation [4-9]. Progressing neuroinflammation, which is a major hallmark of the secondary injury, is mainly initiated through activation of astrocytes and microglia, which are key cells in the maintenance of homeostasis in the CNS, and further boosted and perpetuated by infiltrated neutrophils and macrophages [10-13]. The activation of astrocytes and microglia, so called astrogliosis and microgliosis, respectively, influences the disease outcome in SCI on various levels $[14,15]$.

Astrocytes are the predominant subtype of glial cells in the CNS. Under physiological conditions, they protect neurons through the uptake of excessive neurotransmitters, i.e. glutamate, maintain the integrity of the blood-brain barrier and participate in synaptic stability, plasticity and reorganization $[16,17]$. When being activated, astrocytes become hypertrophic and develop extended processes [6]. Reactive astrocytes are a central component of the glial scar which is formed around the injury site in the secondary injury phase [18]. Glial scar formation affects the healing process and can remain chronically for up to several decades in patients who suffered from SCI [14]. The glial scar limits the spread of inflammation but, at the same time, impedes axonal regeneration [19-21]. Under pathological conditions such as traumatic $\mathrm{SCl}$, reactive astrocytes promote cytotoxic edema formation and ischemia through an upregulation of aquaporin 4 [16]. Further, they are an integral component of local immune responses by producing and secreting a wide range of cytokines and chemokines $[22,23]$. It has been shown that the phenotype of 
reactive astrocytes varies, and it has been assumed that astrocytes can differentiate either in the direction of a more pro-inflammatory A1 or a more anti-inflammatory A2 polarization state [24]. A1 polarized astrocytes express pro-inflammatory cytokines and contribute to neuronal death, whereas A2 polarized astrocytes stimulate CNS recovery and repair $[24,25]$.

The neuroinflammation in $\mathrm{SCl}$ is regulated by expression of pro-inflammatory and anti-inflammatory cytokines, chemokines and other mediators, which are mainly synthesized by glial cells. The glycoprotein lipocalin 2 ( $\mathrm{LCn} 2)$ is considered as a key mediator of immune responses in general and particularly in neurodegenerative diseases [26-31]. It has been shown that Lcn2, which is upregulated at the lesion site of the SC, is produced by astrocytes after $\mathrm{SCl}$ [26]. Further, Lcn2 deficient mice reveal better functional outcomes, a lower expression of chemokines and a reduced extent of secondary injury after SCl in comparison to wild type mice [26]. In general terms, Lcn2 plays an important role in iron transport and homeostasis and promotes the defense against bacterial infections [27, 32]. Furthermore, it has been demonstrated in vitro that Lcn2 has toxic effects on neurons and regulates the expression of proinflammatory cytokines and chemokines [30,33]. It has further been stated that Lcn2 promotes the shifting of the polarization of microglia and astrocytes towards pro-inflammatory phenotypes in vitro [25, 34].

It has been shown that $\mathrm{SCl}$ causes pathological processes in various parts of the body, which were not directly affected by the injury. In patients suffering from SCl cognitive dysfunction, inflammation associated neurodegeneration of brain tissue and an impaired functional brain recovery are commonly observed [35-37]. In addition to the interaction between different parts of the CNS, a further important issue related to neural injury is the communication with peripheral organ systems, i.e. "CNS-organ crosstalk". There are preliminary findings which show that in SCl a defined communication axis exists between $\mathrm{SC}$ and liver suggesting that the liver might exhibit mechanisms that influence neuroinflammation in the SC $[38,39]$.

Due to the limited treatment options in $\mathrm{SCl}$, it is important to identify new possible drug targets. As we suggest Lcn2 to influence SCl pathology, it is of interest to examine its effects on astrocytes, which play a central role in SCl pathology. Additionally, we wanted to get a first impression of whether Lcn2 might participate in the systemic effects of SCI. In the present study, we have analyzed the time course of local Lcn2 expression post SCl and its influence on the activation and polarization of astrocytes. Further, since Lcn2 is also secreted in a paracrine and endocrine fashion, we analyzed the Lcn2 expression in blood stream and other peripheral organs post SCl.

\section{Materials \& Methods}

Animals

We used male C57BL/6J wild type mice (WT) and mice carrying a general Lcn2 deficiency $\left(\mathrm{Lcn}^{-/-}\right)$at the age of 8-14 weeks. The mice were housed and handled in accordance with the guidelines of the 
Federation for European Laboratory Animal Science Associations (FELASA) under standard laboratory conditions. The procedures were approved by the Review Board for the Care of Animal Subjects of the district government (North Rhine-Westphalia, Germany) and performed according to international guidelines on the use of laboratory mice (Az 81-02.04.2018.A227). The WT mice were received from Janvier Labs (Saint-Berthevin Cedex, France). Lcn2 ${ }^{-/-}$mice were bred at the resident Institute for Laboratory Animal Science.

Spinal cord injury

General anesthesia was initiated with isoflurane (2-3 vol\%) in an anesthetic chamber. During surgery, isoflurane (1.5-2 vol\%) was further administered via a face mask. Intraoperative analgesia was attained through injection of buprenorphine (0.05-1 mg/kg s.c.) 30 min preoperatively. After the exposure of the spinal column (T7-T10), a laminectomy of T8 was performed. A standardized injury of the SC at this level was induced by contusion (Infinite Horizons Spinal Cord Impactor) with a force of $60 \mathrm{kdyn}$. After inducing the $\mathrm{SCl}$, the surgical site was sutured in layers and the mice were injected subcutaneously with sterile saline. Postoperative care involved the daily manual emptying of the bladder until spontaneous urination returned. In the control group, only a laminectomy without contusion of the SC was carried out to preclude possible falsifications of the results caused by the mere surgical procedure.

\section{BBB scoring}

To assess functional recovery and locomotion deficits after $\mathrm{SCl}$, the mice were scored in an open field according to Basso, Beattie and Bresnahan (BBB) locomotion rating scale of 0 (complete paralysis) to 21 (normal) as previously described [40]. The scale assesses hind limb movements, body weight support, forelimb to hind limb coordination and whole-body movements.

Tissue preparation

At defined time points after $\operatorname{SCl}(6,12,24,72 \mathrm{~h}$ and $7 \mathrm{~d})$, the mice were transcardially perfused with icecold PBS for molecular biological and protein biochemical studies. The sham operated mice, which served as control, were finalized after $24 \mathrm{~h}$. The whole SC was prepared and divided into three parts of equal size, in the following referred to as rostral, central (lesion site) and caudal region. In addition, the motor and sensory cortex and left liver lobe was prepared. The tissues were immediately snap frozen in liquid nitrogen and kept at $-80^{\circ} \mathrm{C}$ until further processing.

For immunohistochemistry, the mice (control, $24 \mathrm{~h}$ and $72 \mathrm{~h}$ ) were transcardially perfused with ice-cold PBS followed by a $3.7 \%$ paraformaldehyde solution (PFA, pH 7.4). For decalcification, spinal columns were incubated in $20 \%$ EDTA (ethylenediaminetetraacetic acid) for $48 \mathrm{~h}$ at $37^{\circ} \mathrm{C}$ prior to paraffin embedding. Tissue specimens were embedded in paraffin (Merck, Darmstadt, Germany), and $5 \mu \mathrm{m}$ paraffin sections were cut. Blood sampling (control, 6, 12, 24, $72 \mathrm{~h}$ and $7 \mathrm{~d}$ ) was performed through retrobulbar sinus puncture. 
Molecular biological analysis

For RNA isolation, the tissues were placed in homogenization tubes containing $1.4 \mathrm{~mm}$ beads. Samples were homogenized at $5,000 \times \mathrm{g}$ for $15 \mathrm{~s}$. RNA was isolated by phenol-chloroform extraction using peqGold RNA TriFast (PeqLab, Erlangen, Germany). Total RNA amount and purity was determined using 260/280 ratios of optical densities (Nanodrop 1000, PeqLab, Erlangen, Germany). cDNA was obtained by reverse transcription using M-MLV reverse transcription (RT)-kit and random hexanucleotide primers (Invitrogen, Carlsbad, USA). Gene expression levels were analyzed with real-time reverse transcription-PCR (Bio-Rad, Feldkirchen, Germany) using SensiMix ${ }^{T M}$ SYBR ${ }^{\circledR}$ \& Fluorescein Kit (Meridian Bioscience, Cincinnati, USA). Primer sequences and individual annealing temperatures are shown in table 1. Results were evaluated using Bio-Rad CFX manager (Bio-Rad, Feldkirchen, Germany) and were normalized to cyclophilin A and Hsp90 as reference genes. The target gene expression was calculated using the $\Delta \Delta \mathrm{Ct}$ method [41].

\section{Protein biochemical analysis}

Sampled tissues were mechanically disrupted in RIPA buffer ( $\mathrm{pH}$ 8.0) supplemented with a protease inhibitor cocktail (Complete Mini, Roche Diagnostics, Grenzach-Wyhlen, Germany). Protein concentrations were determined using the PierceTM BCA Protein Assay kit (Thermo Fisher Scientific, Waltham, USA) according to the manufacturer's protocol. Per sample, a total of $20 \mu \mathrm{g}$ protein was separated in a $14 \%$ SDS polyacrylamide gel by gel electrophoresis and transferred to a PVDF (polyvinylidene difluoride) membrane. The blots were blocked in $5 \%$ milk in Tris-buffered saline (TBS, pH 7.4) and then incubated overnight (at $4^{\circ} \mathrm{C}$ ) in primary antibodies rabbit anti-Lcn2 in $5 \%$ milk and rabbit anti-GAPDH in $5 \%$ milk (used antibodies are listed in table 2). An appropriate secondary antibody (goat anti-rabbit lgG $(\mathrm{H}+\mathrm{L}$ )HRP) was applied for $2 \mathrm{~h}$ (RT). Signals were analyzed via chemiluminescence detection (Westar Supernova, XLS 3,0100, Cyanagen, Bologna, Italy), visualized (Fusion Solo X, Vilber, Eberhardzell, Germany) and subjected to densitometry analysis using Image J. Results were normalized to GAPDH as reference protein.

\section{ELISA}

Concentrations of Lcn2 in serum were assessed using mouse Lcn2/NGAL Quantikine ELISA Kit (R\&D Systems, Minneapolis, USA) according to the manufacturer's protocol. Samples were assayed in duplicates and used in a 200-fold (control), respectively 500-fold $(6,12,24,72 \mathrm{~h}$ and $7 \mathrm{~d}$ ) dilution. Absorbance was measured using a microplate reader (Tecan $\mathrm{GmbH}$, Männedorf, Switzerland). Final concentrations were calculated from a standard curve.

Immunohistochemistry

For immunohistochemistry (IHC), $5 \mu \mathrm{m}$ thick sections of SC, brain and liver were rehydrated, and antigens were unmasked by heating in Tris/ EDTA ( $\mathrm{pH} 9.0$ ) buffer for $20 \mathrm{~min}$. After blocking with $5 \%$ normal goat serum in PBS, the sections were incubated overnight $\left(4^{\circ} \mathrm{C}\right)$ with rabbit anti-Lcn2 diluted in $5 \%$ normal serum in PBS. Slides were incubated for $30 \mathrm{~min}$ in $0,3 \% \mathrm{H}_{2} \mathrm{O}_{2}$ (in PBS) followed by incubation with goat 
anti- rabbit IgG (H\&L) diluted in 5\% normal serum in PBS for $1 \mathrm{~h}(\mathrm{RT})$. Afterwards, an incubation with ABCsolution (both parts 1:50, VECTASTAIN Elite ABC Kit (Standard), Vector Labs, Burlingame, USA) diluted in PBS for $1 \mathrm{~h}$ (RT) followed.

For double immunofluorescence labeling, sections were blocked with IFF-buffer, containing BSA, FCS and $1 \mathrm{x}$ PBS, for $1 \mathrm{~h}$ and incubated overnight $\left(4^{\circ} \mathrm{C}\right)$ with rabbit anti-Lcn2 diluted in IFF-buffer. The slides were incubated with donkey anti-rabbit 488 diluted in IFF buffer for $1 \mathrm{~h}$ (RT) followed by an incubation with goat anti-GFAP, respectively mouse anti-lba 1 or rat anti-CD31 diluted in IFF buffer overnight $\left(4^{\circ} \mathrm{C}\right)$. Finally, the slides were incubated with donkey anti-goat 594, respectively donkey anti-mouse 594 or goat anti-rat 555 in IFF buffer for $1 \mathrm{~h}(\mathrm{RT})$.

\section{Statistical Analysis}

A total of 55 WT animals were used for the experiments containing 43 animals for qPCR analysis. 24 out of the 43 animals were also used for Western Blot analysis. Samples from 39 animals were subjected for ELISA. For immunohistochemistry staining, we used slices from 12 animals. A total of $20 \mathrm{Lcn}^{-/-}$mice were used for qPCR analysis.

GraphPad Prism 8 (GraphPad Software Inc., San Diego, USA) was used for statistical analysis. BrownForsythe test was performed to test for equal variances and normal distribution was tested with ShapiroWilk test. If necessary, data were transformed via Boxcox for homoscedasticity. One-way ANOVA followed by Dunnett post-hoc test or two-way ANOVA followed by Tukey post-hoc test was used for parametric data. Non-parametric data (Lcn2 mRNA in sensory and motor cortex and Lcn2 concentration in blood serum) were analyzed with Kruskal-Wallis test followed by Dunn's multiple comparisons. WT and Lcn2 ${ }^{-/-}$ data from BBB scoring were compared by an unpaired t-test. All data are given as arithmetic means \pm standard errors of the mean (SEM). The $p$ values were set as ${ }^{*} p \leq 0.05,{ }^{*} p \leq 0.01$, ${ }^{* \star} p \leq 0.001$, and $\star \star \star \star p ~ \leq 0.0001$.

\section{Results}

In a first set of experiments, we aimed at investigating whether traumatic SCl leads to an increase in Lcn2 expression within the $\mathrm{SC}$ and other peripheral organs. Figure 1 shows a significant and stepwise increase of Lcn2 in the central region (injury site) of the SC. mRNA expression immediately rose within the first $6 \mathrm{~h}$ post injury reaching maximum level at $24 \mathrm{~h}$ post $\mathrm{SCl}$ and then rapidly declined at $7 \mathrm{~d}$ (Fig. 1a). Lcn2 protein levels, which were examined by Western Blot, revealed a similar time course and profile with a short delay compared to mRNA expression, peaking at $72 \mathrm{~h}$ post SCl (Figs. 1b/c). To investigate the distribution and localization of Lcn2 positive cells in injured SC, immunostaining against Lcn2 was performed. Immunohistochemistry showed high numbers of Lcn2-positive cells, especially in the gray matter, in the central lesion region $24 \mathrm{~h}$ post injury compared to the control group (Figs. 1d/e/f). Double immunofluorescence staining revealed that Lcn2 signals are associated with GFAP-positive astrocytes (Figs. 1h/i). Iba1-positive microglia (Fig. 1g) did not co-localize with Lcn2 staining in the SC. To determine whether Lcn2 is upregulated in other parts of the SC, we measured the mRNA levels of this molecule in 
rostral and caudal parts. The results indicate a massive upregulation of Lcn2 during the initial $6 \mathrm{~h}$ in the rostral part which persists until $72 \mathrm{~h}$ (Fig. 2a). In the caudal area, we observed a steady upregulation of Lcn2 during the first 7 days post SCl (Fig. 2b).

In a next step, we have examined whether Lcn2 is upregulated in the brain post SCl. In both examined brain regions, sensory and motor cortex, significantly elevated Lcn2 mRNA levels were already present $6 \mathrm{~h}$ post $\mathrm{SCl}$ and thereafter declined (Figs. 2c/d). Protein levels were analyzed in the sensory cortex (Figs. 2e/f) showing a similar expression pattern as Lcn2 mRNA. As shown in Fig. 2, our results from immunofluorescence staining against $L \mathrm{cn} 2$ revealed no reactivity in the brain slices of sham operated mice, but Lcn 2 + cells occurred, mainly around vessels, after SCl (Figs. 2g/h). To identify these cells as endothelial cells, we performed immunofluorescence double staining, which showed a clear colocalization of Lcn2 with the endothelial marker CD31 (Fig. 2i).

Further we analyzed the Lcn2 concentration in blood serum via ELISA, which was significantly elevated around 12 and $24 \mathrm{~h}$ post SCl, reaching a 19-fold increase at its peak (Fig. 3a). In addition, we assessed a potential Lcn2-upregulation in the liver. Here, Lcn2 mRNA (Fig. 3b) and protein (Figs. 3c/d) were significantly elevated from $6 \mathrm{~h}$ post $\mathrm{SCl}$ on, reaching a peak at $12 \mathrm{~h}$ and decreasing again from then on. After immunofluorescence staining almost no Lcn2-immunoreactive cells could be seen in the control group, whereas scattered immunoreaction was detectable after SCI (Figs. 3e/f).

A common phenomenon after $\mathrm{SCl}$ is astrogliosis. Since astrocytes are one of the Lcn2 expressing cell types, we aimed to corelate the expression of astroglial markers (GFAP, vimentin, serpina3n) and Lcn2 in the central SC region (supplementary figures a-c). Like Lcn2 mRNA, GFAP, vimentin and serpina3n mRNA show a significant and progressive increase from $6 \mathrm{~h}$ post SCl on. Serpina3n, like Lcn2, reaches its peak at $24 \mathrm{~h}$ and decreases from then on, whereas GFAP and vimentin levels proceed to rise.

In order to understand the influence of Lcn2 on the pathological scenario after $\mathrm{SCl}$ better, we have included animals with a general Lcn2 deficiency $\left(\mathrm{Lcn} 2^{-/-}\right)$in our study. By comparing results from WT and $\mathrm{LCn}^{-/-}$tissues, we could first demonstrate that the gene expression of the astrogliosis marker GFAP was reduced in the central SC region of $\mathrm{Lcn}^{-/-}$animals compared to WT at all examined time points, with a significant difference between the two genotypes at $24 \mathrm{~h}$ (Fig. 4a).

Activated astrocytes can differentiate in the direction of a more pro- or a more anti-inflammatory state and consequently have varying effects on disease pathology. Since Lcn2 was shown to influence this polarization in vitro, we also addressed the influence of Lcn2 on astrocyte polarization in SCl. The functional polarization of astrocytes is well-acknowledged, with complement component 3 (C3) and sphingosine kinase 1 (SPHK1) as markers for A1 and A2, respectively [24, 42]. Therefore, we analyzed the gene expression profiles of these markers in the injured SC (Figs. 4b-e). In WT mice, we noted that the C3/SPHK1 (A1/A2) quotient was significantly reduced during the first $24 \mathrm{~h}$ after $\mathrm{SCl}$ in the central region and then turned round after $72 \mathrm{~h}$ to become significantly increased (Fig. $4 \mathrm{~b}$ ). This suggests that there are changes in the polarization of astrocytes after SCl. In order to explore whether Lcn2 influences, 
additionally to the extent of astrogliosis, also the functional polarization of astrocytes, we assessed the mRNA expression of the $\mathrm{A} 1$ and $\mathrm{A} 2$ markers stated above in $\mathrm{Lcn} 2^{-/-}$mice. In the central region of the SC, a significant decrease in $A 1$ and $A 2$ marker mRNA could be seen at $24 \mathrm{~h}$ and $7 \mathrm{~d}(\mathrm{~A} 1)$, respectively at all examined time points (A2) in Lcn2 ${ }^{-/-}$mice (Figs. 4c/d). The A1/A2 quotient shows the same pattern as in WT mice with an initial decrease at $24 \mathrm{~h}$ followed by a subsequent increase (Fig. $4 \mathrm{e}$ ).

To assess the effects of $\mathrm{Lcn} 2$ on apoptosis rates, the ratio of BAX mRNA, an apoptotic marker, and Bcl2 mRNA, an anti-apoptotic marker, was evaluated in WT and $\mathrm{Lcn}^{-/-}$mice. As we expected, we observed a significant increase of $\mathrm{BAX} / \mathrm{Bcl} 2$, indicating a pro-apoptotic state, in the central part of the $\mathrm{SC}$ at 24 and $72 \mathrm{~h}$ in WT mice (Fig. 4f). In contrast, the BAX/Bcl2 quotient did not change significantly compared to the control in the rostral and caudal region (supplementary figures $\mathrm{d} / \mathrm{e}$ ). In $\mathrm{Lcn} 2^{-/-}$mice, we observed only a slight reduction of $\mathrm{BAX} / \mathrm{Bcl} 2$ ratios in the central $\mathrm{SC}$ region in comparison to $\mathrm{WT}$, which did not reach a significant level (Fig. 4f).

To assess locomotor impairment and recovery of $\mathrm{WT}$ and $\mathrm{Lcn} 2^{-/-}$mice after $\mathrm{SCl}$, we used BBB scoring (Fig. $4 \mathrm{~g}$ ). Control animals of both genotypes were all rated with a score of 21, demonstrating their unimpaired condition. After $7 \mathrm{~d} \mathrm{Lcn} 2^{-/-}$mice reached a mean score of $\sim 8$, indicating sweeping with no weight support or plantar placement of the paw with no weight support, whereas the mean score of $\sim 4$ in WT mice stands for only slight movement of all three joints of the hindlimbs. The significantly higher scores of $\mathrm{Lcn}^{-/-}$mice at $7 \mathrm{~d}$, indicate better locomotor recovery compared to WT.

\section{Discussion}

In the present study, we used a well-established SCl contusion mouse model to provide evidence that Lcn2 is upregulated after $\mathrm{SCl}$ throughout the whole $\mathrm{SC}$ and not only in the primarily injured region. Beyond SC, we observed a Lcn2-induction in the cerebral cortex at both protein and mRNA level. Interestingly, we show a marked increase of Lcn2 in systemic circulation and also in liver in the early phase post SCl. Various studies have found a correlation between increased Lcn2 levels and CNS disorders, such as multiple sclerosis and stroke $[28,30,43,44]$. Therefore, using Lcn $2^{-/-}$mice, we investigate the effect of Lcn2 deficiency on astrogliosis as a hallmark of SCl. Since the results show a significant reduction of GFAP, a decrease of astrogliosis in Lcn2 deficient mice might be concluded.

Post $\mathrm{SCl}$, astrocytes proliferate and undergo morphological changes which include hypertrophy and the development of extended processes $[6,45]$. Through the release of neurotrophic factors, astrocytes support neurons in $\mathrm{SC}$ and thus, impaired astrocytic function has major consequences for neuronal function $[17,46]$. In brain injury, the ablation of reactive astrocytes was found to lead to substantial neuronal degeneration [17]. Moreover, astrocytes limit the spread of inflammation after SCl, since they are one of the dominant cell types of the glial scar which forms after injury [21, 45]. Furthermore, activated astrocytes can express a variety of cytokines, chemokines, and the respective receptors, and therefore play a pivotal role in the neuroinflammatory processes in $\mathrm{SCl}[45,47]$. Further, axonal regeneration is inhibited by the glial scar and chondroitin sulfate proteoglycans which are produced by reactive glial 
cells, including astrocytes $[19,21]$. In addition, these proteoglycans impede process outgrowth of oligodendrocytes and thereby disturb remyelination $[48,49]$. Based on the dual character of astrocytes, it has been suggested that they can be classified into a neurotoxic $A 1$ and neuroprotective $A 2$ phenotype $[24,25]$. Different factors, such as chemokines and cytokines, e.g. IL-1 $\beta$, TNF- $\alpha$ and IL-10, have been found to control the development of astrocytes in the direction of either phenotype [25,50,51]. One of the regulators of astrocyte polarization is Lcn2 which supports the pro-inflammatory A1 phenotype and decreases the polarization in the direction of A2 in vitro by inhibiting IL-4-STAT6 signaling [25]. The influence of Lcn2 on astrocyte polarization, morphology and migration is an important aspect of its regulatory function in neuroinflammation [27, 52]. Lcn2 is involved in various pathological processes, such as stroke, metabolic inflammation, diabetes and nonalcoholic steatohepatitis [30, 31, 53, 54]. It promotes inflammation through induction of pro-inflammatory cytokines via release of high mobility group box 1, which binds to toll-like receptor 4 and induces oxidative stress by activation of NOX-2 signaling [53]. Furthermore, beyond its effect on activation and polarization of microglia, Lcn2 supports the recruitment of inflammatory cells by the induction of CXCL10 secretion and release of the neutrophilrecruitment signal IL-8 [31, 34, 55-57].

In the present study, we could demonstrate that SCl induces an increase of Lcn2 expression throughout the whole SC. As the cellular source of Lcn2 in the CNS, previous studies have identified astrocytes and endothelial cells, which we confirm by our studies $[26,58]$. However, we could not prove the production of Lcn2 by microglia in our animal model [59]. The triggers of Lcn2 production in this context are, besides others, cytokines such as IL-6 and NF-kappa B activation $[58,60]$.

Since Lcn2 is secreted, and elevated concentrations can be found in the blood circulation under pathological conditions, like multiple sclerosis, intestinal inflammation and arthritic diseases, it has been described as a biomarker in several pathologies [61]. In the present study, we show that the Lcn2 concentration is significantly increased in the serum as a direct consequence of $\mathrm{SCl}$, which might suggest this molecule as a potential biomarker for traumatic SCl. Further, circulating Lcn2 could be considered as a part of the systemic inflammatory response (SIR) which affects the homeostasis of peripheral organs such as liver, kidney, lung and intestine. Thereby it contributes to the pathogenesis of multiple organ dysfunction after $\mathrm{SCl}$ and supports secondary injury to the SC [38, 62-65]. In addition, we were able to detect elevated Lcn2 levels in brain and liver. This can have at least two reasons: Lcn2 might be produced in the respective tissue. This is supported by the fact that we have found significantly increased Lcn2 mRNA in both, brain and liver. Additionally, the identification of Lcn $2+$ cells in both tissues after IHC staining indicates a production of Lcn2 by the resident cells. In the brain, we could identify endothelial cells as a cellular source of $L c n 2$ by double-immunofluorescence staining. One of the possible triggers of Lcn2 production in the brain are cytokines. For example, the i.p. application of IL- 6 induces Lcn2 production by vascular cells in the brain in mice [58]. In adipocytes also TNFa and IL-1 $\beta$ trigger Lcn2 production in vitro [66]. Since various cytokines have been shown to be upregulated in the blood stream after $\mathrm{SCl}$, they might lead to an increase in Lcn2 production in endothelial cells [67]. 
In the liver, hepatocytes and neutrophil granulocytes have been identified as cellular sources of Lcn2 [68, 69]. It has been demonstrated in vitro that the cytokine IL-1 $\beta$ induces Lcn 2 production in a NF-kappa Bdependent manner in both cell types [70-72]. Due to the structure of the hepatic tissue, hepatocytes and recruited neutrophils come into close contact with cytokines, reaching the liver via the hepatic artery which might induce Lcn2 production [73]. Since we have found elevated Lcn2 levels in serum post SCl, Lcn2 might also, besides its production by resident cells, reach the brain and the liver via the bloodstream.

In the brain, Lcn2 has different beneficial as well as harmful effects [74]. In the ischemic brain, Lcn2 contributes to neuronal cell death by promoting neuroinflammation [75]. However, in an experimental model of multiple sclerosis, Lcn2-deficient mice exhibited increased disease severity, suggesting a neuroprotective role of Lcn2 [44]. In liver pathology, the effects of Lcn2 have been discussed controversially. In phases of acute liver injury, Lcn2 plays an essential role in liver homeostasis and lipid metabolism and protects hepatocytes, whereas it promotes liver injury and hepatic steatosis in a model of alcoholic steatohepatitis [76-78].

In our studies, the decrease of the astrogliosis marker GFAP in Lcn $2^{-/-}$mice is a first, valuable hint at a possible promotion of astrogliosis by Lcn2 in SCI [79]. In vitro, it has already been demonstrated that GFAP expression is promoted by Lcn2 [80]. However, according to our results, Lcn2 does not affect the regulatory mechanism underlying the phenotypic polarization of activated astrocytes in our animal model. The promotion of the classical inflammatory activation of astrocytes by Lcn2 has, up to now, been only confirmed in vitro and in an animal model of transient middle cerebral artery occlusion $[25,81]$. Eventually the effect of Lcn2 on astrocyte polarization depends on the underlying pathology.

So far, we base our conclusions regarding the influence of Lcn2 on astrogliosis and astrocyte polarization on qPCR studies. Therefore, possible posttranslational modifications cannot be taken into account. This limitation has to be addressed in further studies. Nevertheless, we confirm a general positive effect of Lcn2 deficiency on the functional outcome in SCI based on BBB locomotor scoring. It is assumed that the elevated level of Lcn2 after SCI may exacerbate axonal degeneration and contribute to poor neurological outcome by enhancing inflammatory cell infiltration and promoting neuronal apoptosis [26].

In summary, we found that SCl promotes the Lcn2-upregulation in SC, brain, blood circulation and peripheral organs such as the liver. Consequently, Lcn2 might play a role in systemic effects and multiple organ dysfunction in $\mathrm{SCl}$ pathology. The precise effect of Lcn2 on peripheral organs has to be examined thoroughly to understand the resulting SCl-induced impairment of these tissues. As a local consequence of SCl pathology, Lcn2 promotes specific aspects of astrogliosis, which suggests that Lcn2 can be therapeutically targeted to modulate the reaction of astrocytes in certain pathologies such as SCl. Further studies are needed to elucidate the precise mechanisms responsible for astrocyte activation and polarization to better understand the role played by Lcn2 in this process.

\section{Declarations}


Acknowledgements We thank Uta Zahn, Petra Ibold and Helga Helten for their technical support.

Funding This project was supported by an internal grant from RWTH Aachen University (A. Zendedel, START- 101/18).

Data Availability The datasets generated and analyzed during this study are available from the corresponding author upon reasonable request.

Code Availability Not applicable.

\section{Authors' information}

Contributions The study was conceptualized and designed by Adib Zendedel, Cordian Beyer, Tim Clarner and Victoria Behrens. Material preparation, data collection and analysis were performed by Victoria Behrens, Weiyi Zhao, Clara Voelz and Nina Müller. The first draft of the manuscript was written by Victoria Behrens and all authors commented on previous versions of the manuscript. All authors read and approved the final manuscript. Adib Zendedel was responsible for the overall supervision of this study.

\section{Compliance with Ethical Standards}

Conflict of interest The authors have no relevant financial or non-financial interests to disclose.

Ethics Approval All animals used in this study were acquired and cared for in accordance with the Federation of European Laboratory Associations (FELASA) recommendations. All experimental procedures and animal care were approved by the Review board for the Care of Animal Subjects of the district government (LANUV, Recklinghausen, North Rhine-Westphalia, Germany).

Consent to Participate Not applicable.

Consent for Publication Not applicable.

\section{References}

1. Westgren, N. and R. Levi, Quality of life and traumatic spinal cord injury. Arch Phys Med Rehabil, 1998. 79(11): p. 1433-9.https://doi.org/10.1016/s0003-9993(98)90240-4

2. Torregrossa, F., M. Salli, and G. Grasso, Emerging Therapeutic Strategies for Traumatic Spinal Cord Injury. World Neurosurg, 2020. 140: p. 591-601.https://doi.org/10.1016/j.wneu.2020.03.199

3. Ahuja, C.S., et al., Traumatic Spinal Cord Injury-Repair and Regeneration. Neurosurgery, 2017. 80(3S): p. S9-S22.https://doi.org/10.1093/neuros/nyw080

4. Fleming, J.C., et al., The cellular inflammatory response in human spinal cords after injury. Brain, 2006. 129(Pt 12): p. 3249-69.https://doi.org/10.1093/brain/awl296

5. Gattlen, C., et al., Spinal Cord T-Cell Infiltration in the Rat Spared Nerve Injury Model: A Time Course Study. Int J Mol Sci, 2016. 17(3): p. 352.https://doi.org/10.3390/ijms17030352 
6. Faulkner, J.R., et al., Reactive astrocytes protect tissue and preserve function after spinal cord injury. J Neurosci, 2004. 24(9): p. 2143-55.https://doi.org/10.1523/JNEUROSCI.3547-03.2004

7. Sekhon, L.H. and M.G. Fehlings, Epidemiology, demographics, and pathophysiology of acute spinal cord injury. Spine (Phila Pa 1976), 2001. 26(24 Suppl): p. S2-12.https://doi.org/10.1097/00007632200112151-00002

8. Ducker, T.B. and D.R. Assenmacher, Microvascular response to experimental spinal cord trauma. Surg Forum, 1969. 20: p. 428-30

9. Guha, A. and C.H. Tator, Acute cardiovascular effects of experimental spinal cord injury. J Trauma, 1988. 28(4): p. 481-90.https://doi.org/10.1097/00005373-198804000-00011

10. Pineau, I., et al., Astrocytes initiate inflammation in the injured mouse spinal cord by promoting the entry of neutrophils and inflammatory monocytes in an IL-1 receptor/MyD88-dependent fashion. Brain Behav Immun, 2010. 24(4): p. 540-53.https://doi.org/10.1016/j.bbi.2009.11.007

11. David, S. and A. Kroner, Repertoire of microglial and macrophage responses after spinal cord injury. Nat Rev Neurosci, 2011. 12(7): p. 388-99.https://doi.org/10.1038/nrn3053

12. Taoka, Y., et al., Role of neutrophils in spinal cord injury in the rat. Neuroscience, 1997. 79(4): p. 117782.https://doi.org/10.1016/s0306-4522(97)00011-0

13. Popovich, P.G., et al., Depletion of hematogenous macrophages promotes partial hindlimb recovery and neuroanatomical repair after experimental spinal cord injury. Exp Neurol, 1999. 158(2): p. 35165.https://doi.org/10.1006/exnr.1999.7118

14. Cregg, J.M., et al., Functional regeneration beyond the glial scar. Exp Neurol, 2014. 253: p. 197207.https://doi.org/10.1016/j.expneurol.2013.12.024

15. Kroner, A. and J. Rosas Almanza, Role of microglia in spinal cord injury. Neurosci Lett, 2019. 709: p. 134370.https://doi.org/10.1016/j.neulet.2019.134370

16. Haydon, P.G. and G. Carmignoto, Astrocyte control of synaptic transmission and neurovascular coupling. Physiol Rev, 2006. 86(3): p. 1009-31.https://doi.org/10.1152/physrev.00049.2005

17. Bush, T.G., et al., Leukocyte infiltration, neuronal degeneration, and neurite outgrowth after ablation of scar-forming, reactive astrocytes in adult transgenic mice. Neuron, 1999. 23(2): p. 297308.https://doi.org/10.1016/s0896-6273(00)80781-3

18. Firkins, S.S., C.A. Bates, and D.J. Stelzner, Corticospinal tract plasticity and astroglial reactivity after cervical spinal injury in the postnatal rat. Exp Neurol, 1993. 120(1): p. 1-

15.https://doi.org/10.1006/exnr.1993.1036

19. Davies, S.J., et al., Regeneration of adult axons in white matter tracts of the central nervous system. Nature, 1997. 390(6661): p. 680-3.https://doi.org/10.1038/37776

20. Okada, S., et al., Conditional ablation of Stat3 or Socs3 discloses a dual role for reactive astrocytes after spinal cord injury. Nat Med, 2006. 12(7): p. 829-34.https://doi.org/10.1038/nm1425

21. Bellver-Landete, V., et al., Microglia are an essential component of the neuroprotective scar that forms after spinal cord injury. Nat Commun, 2019. 10(1): p. 518.https://doi.org/10.1038/s41467-019- 
08446-0

22. Zamanian, J.L., et al., Genomic analysis of reactive astrogliosis. J Neurosci, 2012. 32(18): p. 6391410.https://doi.org/10.1523/JNEUROSCI.6221-11.2012

23. Brambilla, R., et al., Inhibition of astroglial nuclear factor kappaB reduces inflammation and improves functional recovery after spinal cord injury. J Exp Med, 2005. 202(1): p. 145-

56.https://doi.org/10.1084/jem.20041918

24. Liddelow, S.A., et al., Neurotoxic reactive astrocytes are induced by activated microglia. Nature, 2017. 541(7638): p. 481-487.https://doi.org/10.1038/nature21029

25. Jang, E., et al., Phenotypic polarization of activated astrocytes: the critical role of lipocalin-2 in the classical inflammatory activation of astrocytes. J Immunol, 2013. 191(10): p. 520419.https://doi.org/10.4049/jimmunol.1301637

26. Rathore, K.I., et al., Lipocalin 2 plays an immunomodulatory role and has detrimental effects after spinal cord injury. J Neurosci, 2011. 31(38): p. 13412-9.https://doi.org/10.1523/jneurosci.011611.2011

27. Flo, T.H., et al., Lipocalin 2 mediates an innate immune response to bacterial infection by sequestrating iron. Nature, 2004. 432(7019): p. 917-21.https://doi.org/10.1038/nature03104

28. Al Nimer, F., et al., Lipocalin-2 is increased in progressive multiple sclerosis and inhibits remyelination. Neurol Neuroimmunol Neuroinflamm, 2016. 3(1): $p$. e191.https://doi.org/10.1212/NXI.0000000000000191

29. Ni, W., et al., Role of lipocalin-2 in brain injury after intracerebral hemorrhage. J Cereb Blood Flow Metab, 2015. 35(9): p. 1454-61.https://doi.org/10.1038/jcbfm.2015.52

30. Ranjbar Taklimie, F., et al., Hypoxia Induces Astrocyte-Derived Lipocalin-2 in Ischemic Stroke. Int J Mol Sci, 2019. 20(6).https://doi.org/10.3390/ijms20061271

31. Moschen, A.R., et al., Lipocalin-2: A Master Mediator of Intestinal and Metabolic Inflammation. Trends Endocrinol Metab, 2017. 28(5): p. 388-397.https://doi.org/10.1016/j.tem.2017.01.003

32. Yang, J., et al., An iron delivery pathway mediated by a lipocalin. Mol Cell, 2002. 10(5): p. 104556.https://doi.org/10.1016/s1097-2765(02)00710-4

33. Bi, F., et al., Reactive astrocytes secrete Icn2 to promote neuron death. Proc Natl Acad Sci U S A, 2013. 110(10): p. 4069-74.https://doi.org/10.1073/pnas.1218497110

34. Jang, E., et al., Secreted protein lipocalin-2 promotes microglial M1 polarization. FASEB J, 2013. 27(3): p. 1176-90.https://doi.org/10.1096/fj.12-222257

35. Wu, J., et al., Spinal cord injury causes brain inflammation associated with cognitive and affective changes: role of cell cycle pathways. J Neurosci, 2014. 34(33): p. 109891006.https://doi.org/10.1523/JNEUROSCI.5110-13.2014

36. Davidoff, G., et al., Cognitive dysfunction and mild closed head injury in traumatic spinal cord injury. Arch Phys Med Rehabil, 1985. 66(8): p. 489-91 
37. Davidoff, G.N., E.J. Roth, and J.S. Richards, Cognitive deficits in spinal cord injury: epidemiology and outcome. Arch Phys Med Rehabil, 1992. 73(3): p. 275-84

38. Campbell, S.J., et al., Central nervous system injury triggers hepatic CC and CXC chemokine expression that is associated with leukocyte mobilization and recruitment to both the central nervous system and the liver. Am J Pathol, 2005. 166(5): p. 1487-97.https://doi.org/10.1016/S00029440(10)62365-6

39. Cheng, R.D., et al., Spinal cord injury causes insulin resistance associated with PI3K signaling pathway in hypothalamus. Neurochem Int, 2020. 140: p.

104839.https://doi.org/10.1016/j.neuint.2020.104839

40. Basso, D.M., M.S. Beattie, and J.C. Bresnahan, $A$ sensitive and reliable locomotor rating scale for open field testing in rats. J Neurotrauma, 1995. 12(1): p. 1-21.https://doi.org/10.1089/neu.1995.12.1

41. Livak, K.J. and T.D. Schmittgen, Analysis of relative gene expression data using real-time quantitative PCR and the 2(-Delta Delta C(T)) Method. Methods, 2001. 25(4): p. 402-

8.https://doi.org/10.1006/meth.2001.1262

42. Neal, M., et al., Prokineticin-2 promotes chemotaxis and alternative A2 reactivity of astrocytes. Glia, 2018. 66(10): p. 2137-2157.https://doi.org/10.1002/glia.23467

43. Dekens, D.W., et al., Neutrophil Gelatinase-Associated Lipocalin and its Receptors in Alzheimer's Disease (AD) Brain Regions: Differential Findings in AD with and without Depression. J Alzheimers Dis, 2017. 55(2): p. 763-776.https://doi.org/10.3233/JAD-160330

44. Berard, J.L., et al., Lipocalin 2 is a novel immune mediator of experimental autoimmune encephalomyelitis pathogenesis and is modulated in multiple sclerosis. Glia, 2012. 60(7): p. 114559.https://doi.org/10.1002/glia.22342

45. O'Shea, T.M., J.E. Burda, and M.V. Sofroniew, Cell biology of spinal cord injury and repair. J Clin Invest, 2017. 127(9): p. 3259-3270.https://doi.org/10.1172/JCI90608

46. Freeman, K.A., et al., Spinal cord protection via alpha-2 agonist-mediated increase in glial cell-linederived neurotrophic factor. J Thorac Cardiovasc Surg, 2015. 149(2): p. 578-84; discussion 5846.https://doi.org/10.1016/j.jtcvs.2014.10.037

47. Sofroniew, M.V., Multiple roles for astrocytes as effectors of cytokines and inflammatory mediators. Neuroscientist, 2014. 20(2): p. 160-72.https://doi.org/10.1177/1073858413504466

48. Siebert, J.R. and D.J. Osterhout, The inhibitory effects of chondroitin sulfate proteoglycans on oligodendrocytes. J Neurochem, 2011. 119(1): p. 176-88.https://doi.org/10.1111/j.14714159.2011.07370.x

49. Tran, A.P., P.M. Warren, and J. Silver, The Biology of Regeneration Failure and Success After Spinal Cord Injury. Physiol Rev, 2018. 98(2): p. 881-917.https://doi.org/10.1152/physrev.00017.2017

50. Liu, L.R., et al., Interaction of Microglia and Astrocytes in the Neurovascular Unit. Front Immunol, 2020. 11: p. 1024.https://doi.org/10.3389/fimmu.2020.01024

51. Hyvarinen, T., et al., Co-stimulation with IL-1beta and TNF-alpha induces an inflammatory reactive astrocyte phenotype with neurosupportive characteristics in a human pluripotent stem cell model 
system. Sci Rep, 2019. 9(1): p. 16944.https://doi.org/10.1038/s41598-019-53414-9

52. Lee, S., M.K. Jha, and K. Suk, Lipocalin-2 in the Inflammatory Activation of Brain Astrocytes. Crit Rev Immunol, 2015. 35(1): p. 77-84.https://doi.org/10.1615/critrevimmunol.2015012127

53. Mondal, A., et al., Lipocalin 2 induces neuroinflammation and blood-brain barrier dysfunction through liver-brain axis in murine model of nonalcoholic steatohepatitis. J Neuroinflammation, 2020. 17(1): p. 201.https://doi.org/10.1186/s12974-020-01876-4

54. Rashad, N.M., et al., Lipocalin-2 expression and serum levels as early predictors of type 2 diabetes mellitus in obese women. IUBMB Life, 2017. 69(2): p. 88-97.https://doi.org/10.1002/iub.1594

55. Shashidharamurthy, R., et al., Differential role of lipocalin 2 during immune complex-mediated acute and chronic inflammation in mice. Arthritis Rheum, 2013. 65(4): p. 1064-

73.https://doi.org/10.1002/art.37840

56. Lee, S., et al., Lipocalin-2 Is a chemokine inducer in the central nervous system: role of chemokine ligand 10 (CXCL10) in lipocalin-2-induced cell migration. J Biol Chem, 2011. 286(51): p. 4385543870.https://doi.org/10.1074/jbc.M111.299248

57. Bachman, M.A., V.L. Miller, and J.N. Weiser, Mucosal lipocalin 2 has pro-inflammatory and ironsequestering effects in response to bacterial enterobactin. PLoS Pathog, 2009. 5(10): p. e1000622.https://doi.org/10.1371/journal.ppat.1000622

58. Hamzic, N., A. Blomqvist, and C. Nilsberth, Immune-induced expression of lipocalin-2 in brain endothelial cells: relationship with interleukin-6, cyclooxygenase-2 and the febrile response. J Neuroendocrinol, 2013. 25(3): p. 271-80.https://doi.org/10.1111/jne.12000

59. Lee, S., et al., A dual role of lipocalin 2 in the apoptosis and deramification of activated microglia. J Immunol, 2007. 179(5): p. 3231-41.https://doi.org/10.4049/jimmunol.179.5.3231

60. Ouali Alami, N., et al., NF-kappaB activation in astrocytes drives a stage-specific beneficial neuroimmunological response in ALS. EMBO J, 2018.

37(16).https://doi.org/10.15252/embj.201798697

61. Abella, V., et al., The potential of lipocalin-2/NGAL as biomarker for inflammatory and metabolic diseases. Biomarkers, 2015. 20(8): p. 565-71.https://doi.org/10.3109/1354750X.2015.1123354

62. Gris, D., E.F. Hamilton, and L.C. Weaver, The systemic inflammatory response after spinal cord injury damages lungs and kidneys. Exp Neurol, 2008. 211(1): p. 259-

70.https://doi.org/10.1016/j.expneurol.2008.01.033

63. Zhang, C., et al., Gut microbiota dysbiosis in male patients with chronic traumatic complete spinal cord injury. J Transl Med, 2018. 16(1): p. 353.https://doi.org/10.1186/s12967-018-1735-9

64. Campbell, I.L., Cytokine-mediated inflammation, tumorigenesis, and disease-associated JAK/STAT/SOCS signaling circuits in the CNS. Brain Res Brain Res Rev, 2005. 48(2): p. 16677.https://doi.org/10.1016/j.brainresrev.2004.12.006

65. Sundman, M.H., et al., The bidirectional gut-brain-microbiota axis as a potential nexus between traumatic brain injury, inflammation, and disease. Brain Behav Immun, 2017. 66: p. 3144.https://doi.org/10.1016/j.bbi.2017.05.009 
66. Zhang, Y., et al., Lipocalin 2 expression and secretion is highly regulated by metabolic stress, cytokines, and nutrients in adipocytes. PLoS One, 2014. 9(5): p. e96997.https://doi.org/10.1371/journal.pone.0096997

67. Mukhamedshina, Y.O., et al., Systemic and Local Cytokine Profile following Spinal Cord Injury in Rats: A Multiplex Analysis. Front Neurol, 2017. 8: p. 581.https://doi.org/10.3389/fneur.2017.00581

68. Molina, L., et al., Hepatocyte-Derived Lipocalin 2 Is a Potential Serum Biomarker Reflecting Tumor Burden in Hepatoblastoma. Am J Pathol, 2018. 188(8): p. 1895-

1909.https://doi.org/10.1016/j.ajpath.2018.05.006

69. Li, H., et al., Hepatocytes and neutrophils cooperatively suppress bacterial infection by differentially regulating lipocalin-2 and neutrophil extracellular traps. Hepatology, 2018. 68(4): p. 16041620.https://doi.org/10.1002/hep.29919

70. Jayaraman, A., et al., Identification of neutrophil gelatinase-associated lipocalin (NGAL) as a discriminatory marker of the hepatocyte-secreted protein response to IL-1beta: a proteomic analysis. Biotechnol Bioeng, 2005. 91(4): p. 502-15.https://doi.org/10.1002/bit.20535

71. Cowland, J.B., et al., Neutrophil gelatinase-associated lipocalin is up-regulated in human epithelial cells by IL-1 beta, but not by TNF-alpha. J Immunol, 2003. 171(12): p. 66309.https://doi.org/10.4049/jimmunol.171.12.6630

72. Borkham-Kamphorst, E., F. Drews, and R. Weiskirchen, Induction of lipocalin-2 expression in acute and chronic experimental liver injury moderated by pro-inflammatory cytokines interleukin-1beta through nuclear factor-kappaB activation. Liver Int, 2011. 31(5): p. 65665.https://doi.org/10.1111/j.1478-3231.2011.02495.x

73. Fleming, J.C., et al., Remote inflammatory response in liver is dependent on the segmental level of spinal cord injury. J Trauma Acute Care Surg, 2012. 72(5): p. 1194-201;discussion 1202.https://doi.org/10.1097/TA.0b013e31824d68bd

74. Ferreira, A.C., et al., From the periphery to the brain: Lipocalin-2, a friend or foe? Prog Neurobiol, 2015. 131: p. 120-36.https://doi.org/10.1016/j.pneurobio.2015.06.005

75. Jin, M., et al., Lipocalin-2 deficiency attenuates neuroinflammation and brain injury after transient middle cerebral artery occlusion in mice. J Cereb Blood Flow Metab, 2014. 34(8): p. 130614.https://doi.org/10.1038/jcbfm.2014.83

76. Borkham-Kamphorst, E., et al., Protective effects of lipocalin-2 (LCN2) in acute liver injury suggest a novel function in liver homeostasis. Biochim Biophys Acta, 2013. 1832(5): p. 66073.https://doi.org/10.1016/j.bbadis.2013.01.014

77. Asimakopoulou, A., et al., Lipocalin-2 (LCN2) regulates PLIN5 expression and intracellular lipid droplet formation in the liver. Biochim Biophys Acta, 2014. 1842(10): p. 151324.https://doi.org/10.1016/j.bbalip.2014.07.017

78. Wieser, V., et al., Lipocalin 2 drives neutrophilic inflammation in alcoholic liver disease. J Hepatol, 2016. 64(4): p. 872-80.https://doi.org/10.1016/j.jhep.2015.11.037 
79. Li, T., et al., An update on reactive astrocytes in chronic pain. J Neuroinflammation, 2019. 16(1): p. 140.https://doi.org/10.1186/s12974-019-1524-2

80. Lee, S., et al., Lipocalin-2 is an autocrine mediator of reactive astrocytosis. J Neurosci, 2009. 29(1): p. 234-49.https://doi.org/10.1523/JNEUROSCI.5273-08.2009

81. Zhao, N., et al., Lipocalin-2 may produce damaging effect after cerebral ischemia by inducing astrocytes classical activation. J Neuroinflammation, 2019. 16(1): p.

168.https://doi.org/10.1186/s12974-019-1556-7

82. Franklin, K.B.J. and G. Paxinos, Paxinos and Franklin's the Mouse Brain in Stereotaxic Coordinates. 2019: Academic Press.

\section{Tables}

Due to technical limitations, table 1 and 2 is only available as a download in the Supplemental Files section.

\section{Figures}
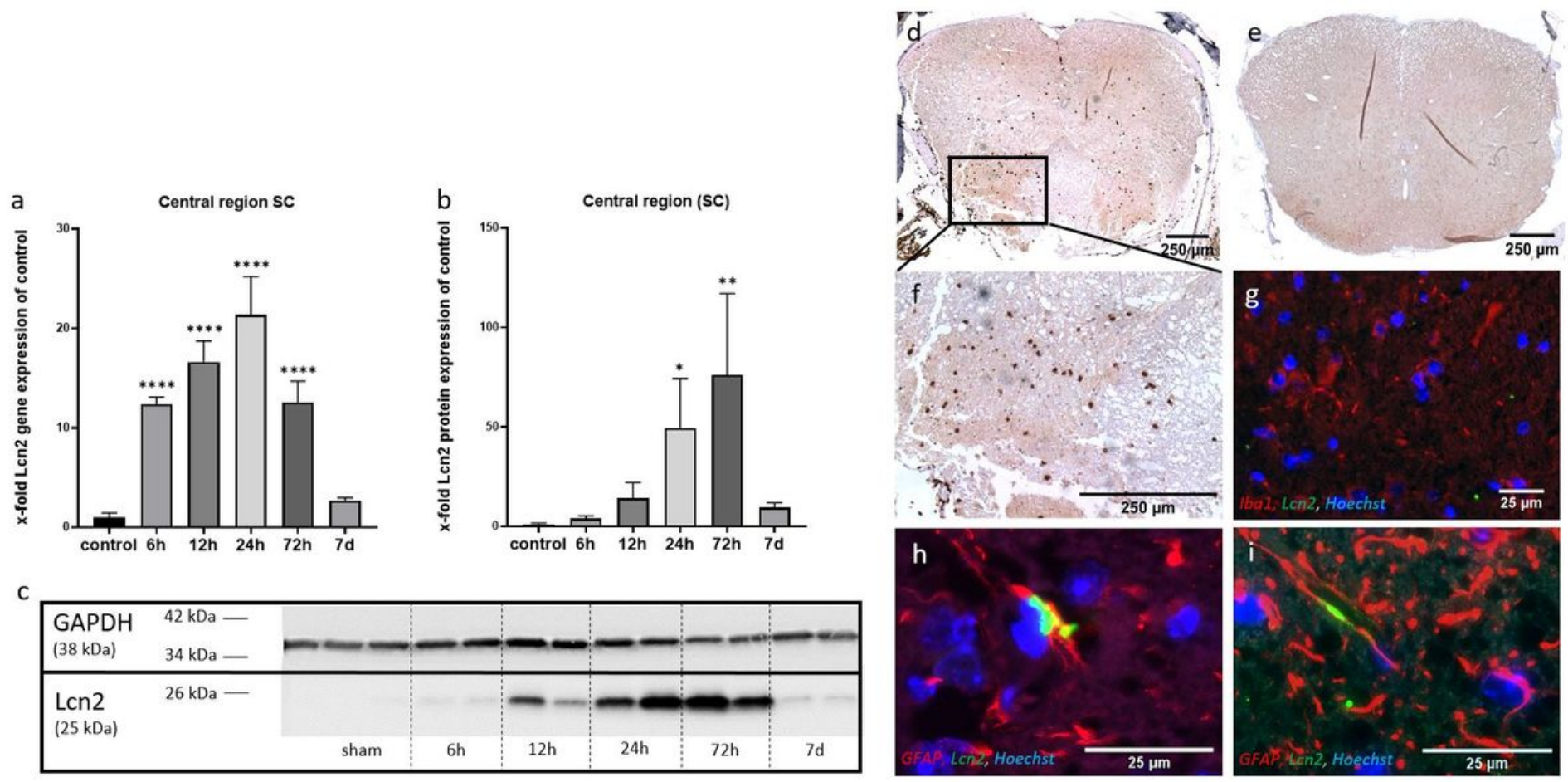

\section{Figure 1}

Lcn2 expression in central region of SC post SCl in WT mice. Lcn2 gene (a) ( $n=6 ; 72 h, 7 d n=5)$ and protein expression $(b / c)(n=4)$. IHC staining against Lcn2 of representative sections of the groups $24 \mathrm{~h}$ (d/f) and control (e). Double immunofluorescence labeling $24 \mathrm{~h}$ post SCI for Lcn2 and Iba1 (g), 
respectively Lcn2 and GFAP (h/i). Data represent means \pm SEM. ${ }^{* \star *} p<<0.0001,{ }^{*} \mathrm{p}<0.01,{ }^{*} \mathrm{p}<0.05$ indicate control vs. time point
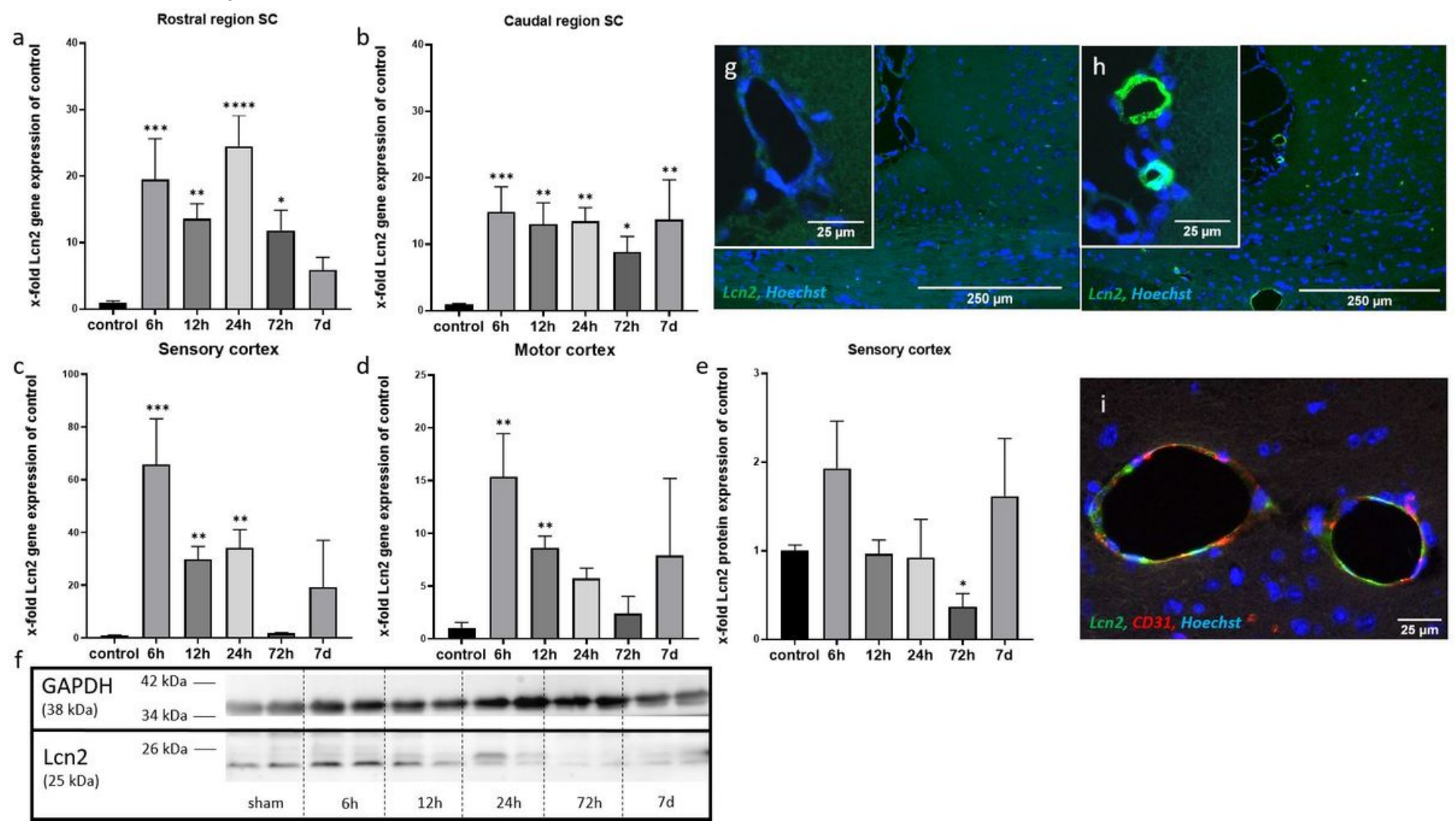

Figure 2

Lcn2 gene expression in rostral (a) $(n=6$; control $n=5)$ and caudal (b) $(n=6 ; 7 d n=5)$ region of SC. Lcn2 gene expression in sensory (c) (control $n=5 ; 6 h, 12 h, 24 h, 7 d n=7 ; 72 h n=8$ ) and motor cortex (d) (control $n=6 ; 6 h n=5 ; 12 h n=8 ; 24 h, 72 h, 7 d n=7)$. Lcn2 protein expression in sensory cortex $(e / f)(n=4)$. Representative brain sections (Bregma - 0,82 mm) [82] from control $(\mathrm{g})$ and $24 \mathrm{~h}$ group $(\mathrm{h})$ after IHC staining against $L c n 2$. Representative brain section after double immunofluorescence labeling $24 \mathrm{~h}$ post SCI for Lcn2 and endothelial marker CD31 (i). Data represent means \pm SEM. ${ }^{* \star * *} p<0.0001,{ }^{\star \star *} p<0.001$, $\star * p<0.01, * p<0.05$ indicate control vs time point 

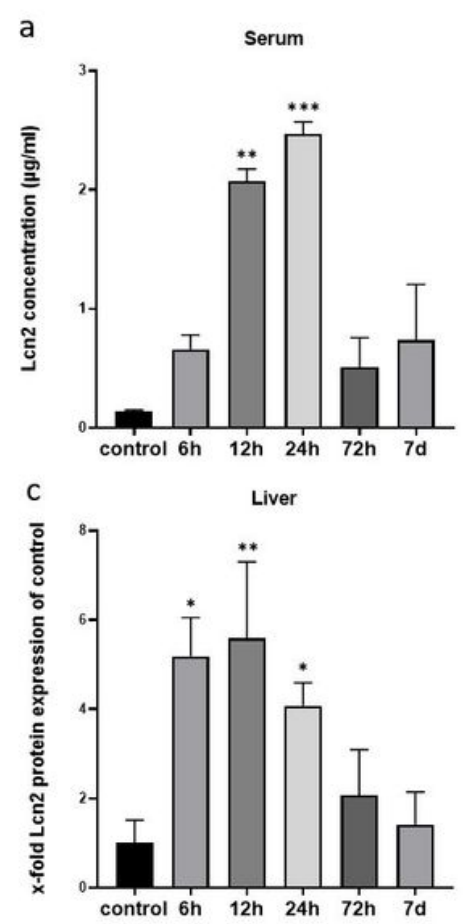
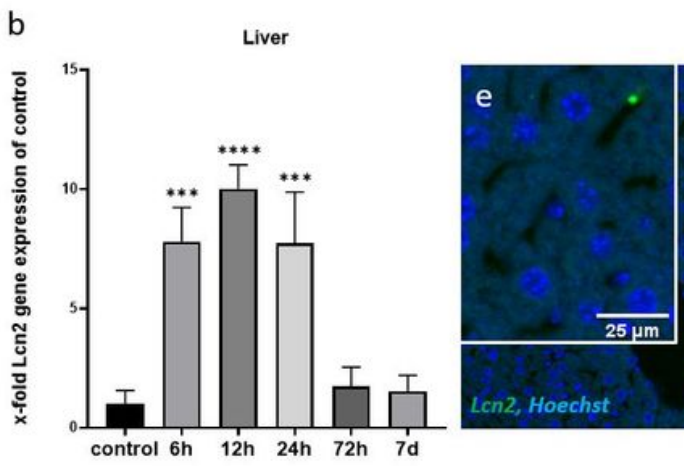

Len2, Hoechst
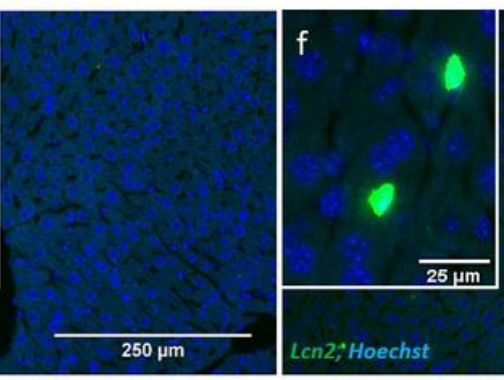

d

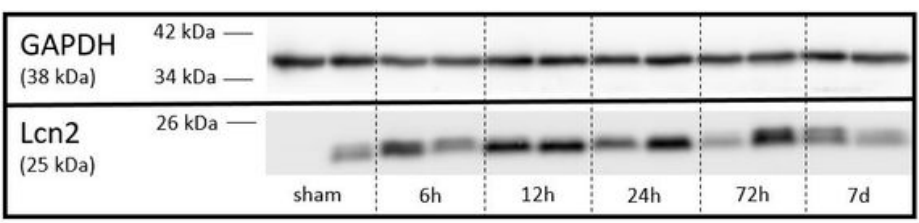

Figure 3

Lcn2 concentration in blood serum (a) (control $n=5 ; 6$ h n=6; 12 h, 24 h, 72 h, 7 d n=7). Lcn2 gene expression in liver (b) $(n=5)$. Lcn2 protein expression in liver $(c / d)(n=4)$. Representative liver sections from control (e) and $72 \mathrm{~h}$ group (f) immunofluorescence labeled for Lcn2. Data represent means \pm SEM. ${ }^{\star * * *} p<0.0001,{ }^{* *} p<0.001,{ }^{* *} p<0.01,{ }^{*} p<0.05$ indicate control vs time point
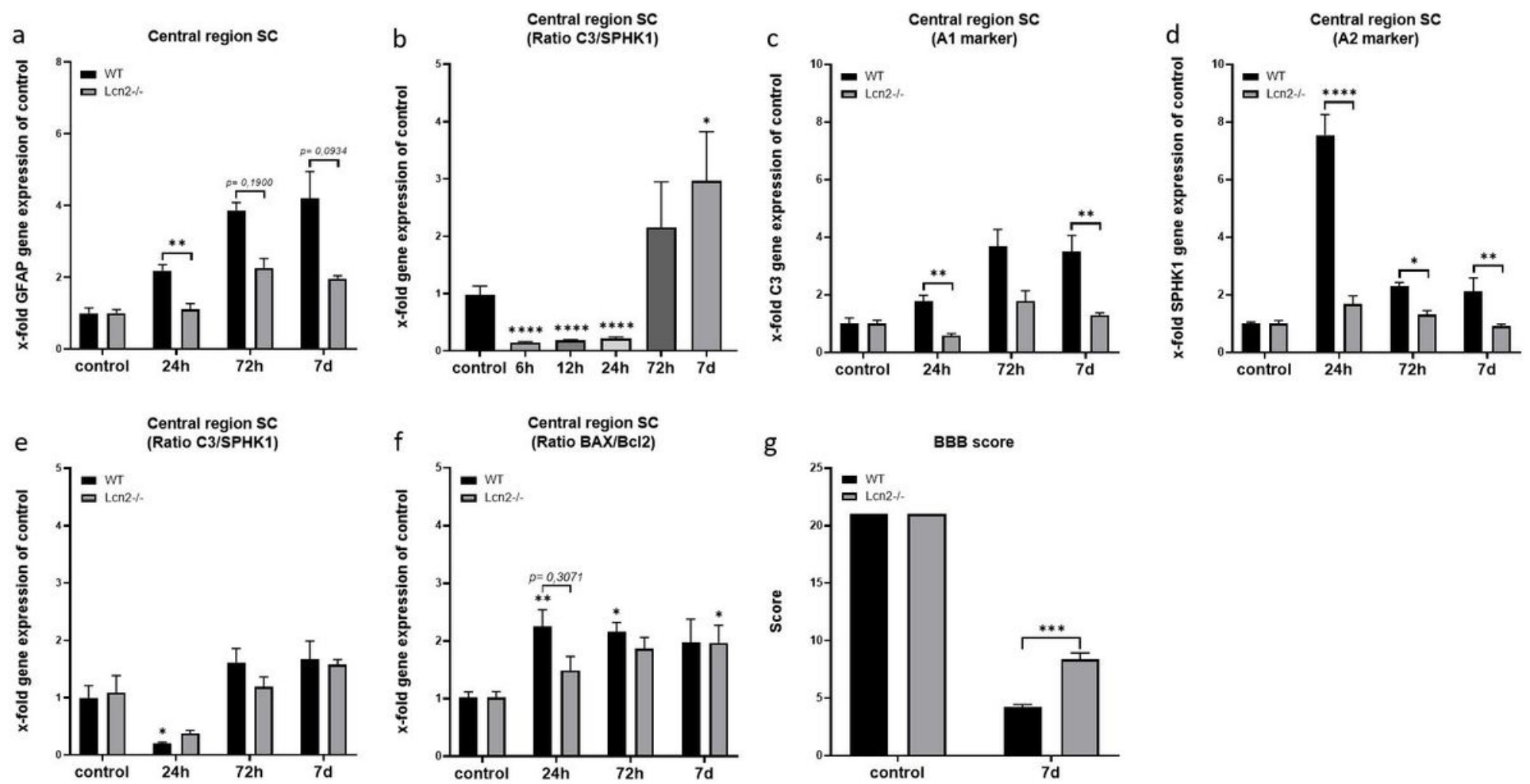


\section{Figure 4}

GFAP gene expression in WT and Lcn2 /- mice in central (a) (n=5) region of SC. Ratio of gene expression of A1 astrocyte marker C3 and A2 astrocyte marker SPHK1 in WT mice (b) ( $n=6)$. Gene expression of C3 (c) $(n=5)$ and SPHK1 (d) (n=5) in WT and Lcn $2 /$ - mice in central region of SC. Ratio of gene expression of C3 and SPHK1 in WT and Lcn2 -/- mice (e) $(n=5)$. Ratio of gene expression of pro-apoptotic marker $B A X$ and anti-apoptotic marker Bcl2 in WT and Lcn $2-/$ - mice in central region of $S C(f)(n=5)$. Comparison of BBB scoring of WT and Lcn2 /- mice $7 \mathrm{~d}$ post SCl (g) (WT n=10; Lcn2 -/- $n=8)$. Data represent means \pm SEM. ${ }^{* \star *}{ }^{*}<0.0001,{ }^{* * *} p<0.001,{ }^{*} \mathrm{p}<0.01,{ }^{*} \mathrm{p}<0.05$ indicate control vs time point $(\mathrm{b} / \mathrm{e} / \mathrm{f})$, respectively WT vs. Lcn $2 /-(a / c / d / g)$

\section{Supplementary Files}

This is a list of supplementary files associated with this preprint. Click to download.

- Supplementaryfigures.pptx

- Table2.pptx

- Table1.pptx 